\title{
50 Jahre Katzenmedizin - ein Rück- und Ausblick
}

\author{
Jürgen Kremendahl
}

Veränderungen in der Medizin sind allgegenwärtig und nötig; das trifft auch für die Tiermedizin zu. In dieser Übersicht wird ein Teilgebiet der Tiermedizin beleuchtet, das in den vergangenen Jahrzehnten eine wahrlich beachtenswerte Entwicklung erfahren hat: die Katzenmedizin.

\section{Einleitung}

Im Jahre 1961 veröffentlichte der Tierarzt J.E.B. Graham in einer kanadischen Zeitschrift für Tierärzte eine „umfassende“ Übersichtsarbeit zum Thema Katzenmedizin [2]. Dafür benötigte er nur wenige Seiten! Mehr als 50 Jahre später hat das medizinische Wissen zur Spezies Katze in unglaublicher Weise zugenommen. Es gibt inzwischen Fachbücher zu allen medizinischen Teilgebieten der Katzenmedizin - ein paar Seiten reichen heute allenfalls zur Darstellung eines interessanten Fallberichts.

Das Interesse an diesem Teilbereich der Kleintiermedizin nahm und nimmt weltweit stark zu. Am „World Feline Veterinary Congress“ im Juni 2013 in Barcelona nahmen beispielsweise 650 Personen aus 37 Nationen teil. Zeugnis dafür, dass dieses Wissen auch in den Praxen ankommt.

Warum sind Katzen also - medizinisch gesehen - nicht mehr die viel zitierten „kleinen Hunde“? Es gibt sicher mehr als einen Anlass, die Katzenmedizin der letzten Jahrzehnte - ohne jegliche Absicht der Vollständigkeit - Revue passieren zu lassen.

\section{Anfänge}

Die veterinärmedizinische Literatur bot zu jeder Zeit interessante Arbeiten zum
Forschungsobjekt Katze, z. B. eine Arbeit über die infektiöse Peritonitis der Katze (FIP) aus dem Italien der 1940er Jahre [1]. Diese darf sicher als eine der ersten Veröffentlichungen zum Thema FIP gelten, eine Krankheit, die uns leider heute nach über 70 Jahren noch genauso beschäftigt wie den Pathologen A. Bonaduce im Kriegsjahr 1942 in Neapel.

Eine wesentliche Rolle bei der Etablierung der Katzenmedizin als eigenständige Disziplin kommt sicher Jean Holzworth, der „Mutter der Katzenmedizin“, zu. Sie prägte die amerikanische Katzenmedizin maßgeblich von 1951 bis zu ihrem Ruhestand im Jahre 1986 im Angell Memorial Animal Hospital in Boston, USA. Auch sie publizierte erste wesentliche Beiträge zum Thema FIP in den frühen 1960er Jahren.

\section{USA}

Es wundert nicht, dass auch die Anfänge der „privaten“ Katzenmedizin in den USA zu finden sind. Anfang der 1970er Jahre eröffneten in etwa zeitgleich die ersten beiden privaten Katzenkliniken:

- das „Cat Hospital of Philadelphia“ (Susan McDonough)

- die „Chicago Cat Clinic“ (Barbara Stein)

Beide Tierärzte hatten zuvor in größeren Kliniken gearbeitet, dort aber fast nur Katzen betreut, um dann den Schritt der Gründung einer reinen Katzenpraxis zu wagen, was in beiden Fällen mit nachhaltigem Erfolg gelang. Beide Kliniken, die in ihrer Gründungskonzeption sehr unterschiedlich waren, existieren heute noch. Beispielweise legte Susan McDonough in Philadelphia viel Wert auf ein gemütliches, fast wohnzimmerartiges Ambiente, während Barbara Stein einer konsequenten Klinikatmosphäre (im Orange der 1970er Jahre) den Vorzug gab. Viele Hunderte Katzenpraxen sind im Laufe der Jahrzehnte in den USA dazu gekommen. Es gibt nur noch wenige Städte in den USA, in denen man vergeblich nach einer solchen Praxis sucht.

\section{Europa}

In Mitteleuropa klafft die Lücke zwischen der „universitären“ und der „privaten“ Katzenmedizin bis zum heutigen Tage weit mehr als in den USA oder auch in Australien.

Auf universitärem Niveau war und ist die Katzenmedizin auch in Europa stets präsent gewesen: Besonders hervorgehoben seien hier die Universitäten in Bristol, Glasgow, Liverpool, Zürich und in Gießen. Die Namen der dort tätigen Dozenten und Forscher (alphabetisch und ohne Anspruch auf Vollständigkeit) sind jedem katzeninteressierten Tierarzt bekannt: Diane Addie, Sue Dawson, Rosalind Gaskell, Tim Gruffydd-Jones, Marian Horzinek, Os Jarrett, Anja Kipar, Hans Lutz, Carmel Mooney, Manfred Reinacher und Andy Sparkes.

Die Gründung privater Katzenpraxen hingegen verlief und verläuft auf dieser Seite des Atlantiks recht schleppend. Auch wenn es schon in den 1990er Jahren vereinzelt Katzen- und Katzenschwerpunktpraxen in Europa gab, konnte man seinerzeit nicht wirklich von einer Entwicklung sprechen. Zwei der Pioniere in dieser Hinsicht waren sicher Michèle Fradin-Fermé (Paris, Frankreich) und David R. Godfrey mit Ehefrau Rosie Anderson (Solihull, West Midlands, Großbritannien). In England war es beispielsweise bereits in den 1990er Jahren - trotz eines sehr strikten Royal College of Veterinary Surgeons - kein Problem, den Namen „Nine Lives Veterinary Practice for Cats“ (in England haben Katzen neun Leben!) zu verwenden. Die Bezeichnung „Katzenpraxis“ ist in manchen anderen europäi- 


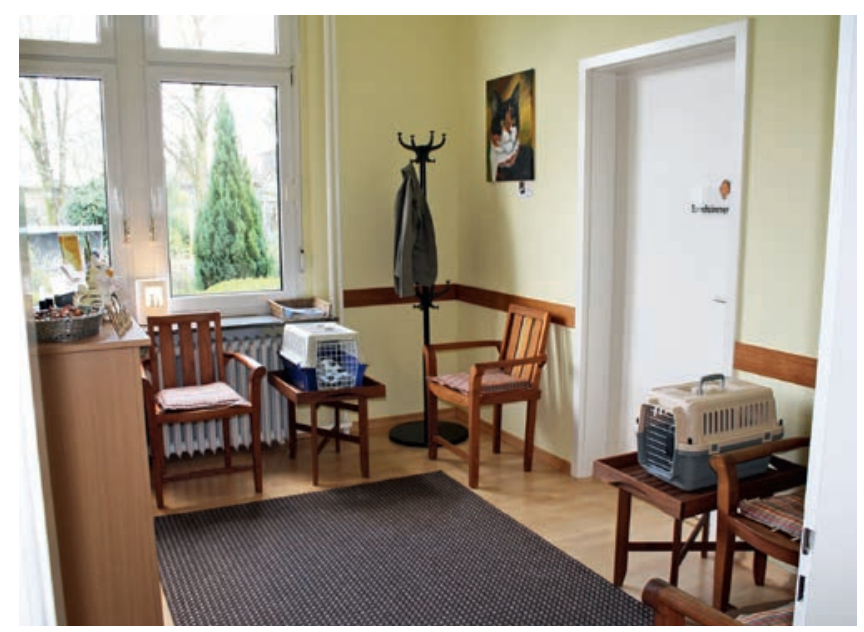

Abb. 1 Rezeption und Teil des Wartebereichs in der Praxis des Verfassers. Durch Farbwahl und Verwendung von (entsprechend zu reinigenden) Textilien wurde versucht, eine „wohnliche“ Atmosphäre zu schaffen. (c) J. Kremendahl

schen Ländern aber nach wie vor problematisch. Zum Teil muss die korrekte Praxisbezeichnung hilfsweise mittels der Zusätze „Schwerpunkt Katzenmedizin“ oder „Sprechstunden nur für Katzen“ hergestellt werden. Der Katzenhalter hat seit jeher diesbezüglich weniger Berührungsängste als manche Kammer: schließlich kennt er auch Pferdekliniken, Koi-Tierärzte und Vogelpraxen.

\section{Warum eine Praxis nur für Katzen?}

Warum eigentlich gibt es weltweit in zunehmendem Maße Katzenpraxen, wo es doch - zumindest nach Kenntnisstand des Verfassers - in der ganzen Welt keine einzige „reine“ Hundepraxis gibt? Neben den witzig-sarkastischen Gründen aus Sicht einiger Hundehalter („Mein Hund langweilt sich in einem Wartezimmer ohne Katzen!") gibt es sicher auch belastbarere Gründe, die im Folgenden genannt werden.

\section{Stress}

Katzen - und das hört der Verfasser auch nach 20 Jahren Katzenpraxis noch fast täglich - mögen eine ruhige Praxis u.a. ohne Gebell und Hundegeruch deutlich lieber. Sie sind weniger gestresst und lassen eher unangenehme Maßnahmen über sich ergehen.

\section{.konkret}

In einer reinen Katzenpraxis sind

Sedationen deutlich seltener nötig.
Es bleiben selbstverständlich genügend Katzen übrig, die - in der Regel infolge einer Angstaggression - nicht wahrnehmbar anders reagieren als in jeder anderen Kleintierpraxis oder -klinik. Daher sind Hände und Arme eines Katzenpraktikers am Ende einer Woche nicht unbedingt von denen eines „allgemeinen“ Kleintierkollegen zu unterscheiden. Viele dieser Effekte sind natürlich auch durch Abteilung eines Katzenbereichs (mit eigener Wartezone und eigenem Sprechzimmer) zu erzielen.

\section{Räumlichkeiten}

Der Raumbedarf einer reinen Katzenpraxis ist naturgemäß geringer, auch müssen die Räumlichkeiten nicht unbedingt im Erdgeschoss liegen. Probleme mit Nachbarn (Kot- und Urinabsatz an der Zuwegung, übermäßiges Bellen etc.) dürften kaum auftreten. Die Inneneinrichtung einer Katzenpraxis lässt - unter Einhaltung moderner hygienischer Standards - sicher ein wenig mehr Spielraum für individuelle Gestaltung ( $\triangleright$ Abb.1). Es schüttelt sich beispielweise kein nasser Hund, wenn es draußen „cats and dogs“ regnet. Katzenbesitzer mögen eine katzengerechte Umgebung, wobei „katzengerecht" Funktion und Geschmack meint. Wer sich derartige Gedanken für bestehende oder zukünftige Räume macht, sollte sich unbedingt mit dem „Cat Friendly Clinic" Programm beschäftigen. Dieser Standard nach einem fest umschriebenen Kriterienkatalog kann auch in Deutschland erworben werden. Leider ist die Anzahl der so „zertifizierten“ Praxen in Deutschland noch sehr gering ( $\bullet$ Abb.2). 


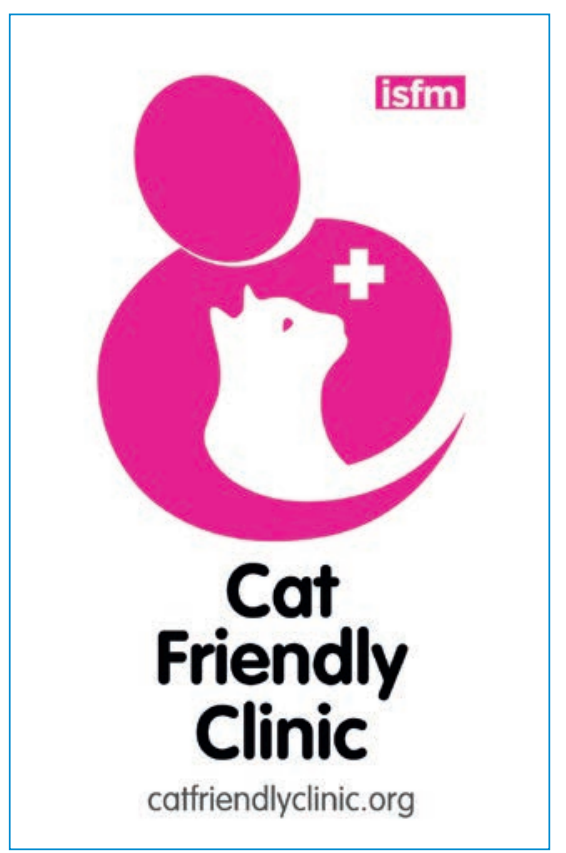

Abb. 2 Das Logo „Cat Friendly Clinic“ erhalten Praxen und Kliniken, die die entsprechenden Kriterien erfüllt haben. (c) International Cat Care

\section{Praxisausrüstung}

Neben der Praxiseinrichtung weicht auch die Ausstattung mit Geräten von der einer allgemeinen Kleintierpraxis ab. Da die Patienten einer Katzenpraxis alle - in etwa - gleich groß sind, ist es nicht erforderlich, z.B. Endotrachealtuben, Boxen für die stationäre Aufnahme etc. in allen Größen vorrätig zu halten. Ein Röntgengerät muss nicht unbedingt besonders leistungsstark sein, um gute Bilder zu erzeugen. Das Ultraschallgerät benötigt „nur“ katzenoptimale Schallköpfe und Endoskope müssen einen angemessen kleinen Durchmesser haben. Man wird angesichts der Zahngesundheit der Katze und des zunehmenden Bewusstseins der Katzenhalter in Bezug auf diese Problematik auf Dauer kaum ohne Dentalröntgen auskommen ( $\triangleright$ Abb.3). Ähnliches gilt für ein von der Fachwelt akzeptiertes Gerät zur Blutdruckmessung ( Abb.4). Kurzum, die Praxisausrüstung ist deutlich weniger breit gefächert, muss aber den speziellen Erfordernissen Rechnung tragen; preiswerter ist sie sicher nicht in jedem Fall.

\section{Motivation}

Nach all diesen Aspekten aus Sicht der Katze, des Halters und der Technik soll nun die Motivation des (zukünftigen) Betreibers einer Katzenpraxis erörtert werden. In den meisten dem Verfasser bekannten „Fällen“ war es eine konsequente Entwicklung. Der katzeninteressierte und katzenhaltende Student macht im Rahmen eines Praktikums erste Erfahrungen mit der „Katzenmedizin“. Er bemüht sich um entsprechende Facharbeiten, Dissertationsthemen, Residencies etc. und rutscht so dauerhaft in dieses Gebiet der Kleintiermedizin.

Auf der anderen Seite gibt es auch Katzenpraxen oder -kliniken, die ausschließlich aus wirtschaftlichen Überlegungen gegründet wurden und die auch ohne ,idealistische Verklärung“ genauso gute $\mathrm{Ar}$ beit leisten. Viele mittelgroße und große Städte „vertragen“ einfach nicht noch eine allgemeine Kleintierpraxis. Wenn man hier niederlassungswillig und ortsgebunden ist, bleibt nur die „Abhebung“ über eine Spezialisierung. Diese kann tierart- spezifisch (Vogel, Reptilien, Katze etc.) oder organspezifisch (Kardiologie, Ethologie, Ophthalmologie etc.) sein. Die Motivation spielt für den (wirtschaftlichen) Erfolg einer Praxis kaum eine Rolle. Ausbildung und Wissen zum Spezialgebiet sowie Patientenkommunikation auf Tierarzt- wie Helferinnenebene sind hingegen bedeutsam.

\section{Qualifikationen}

Es erscheint kaum realistisch, in Deutschland auf die Möglichkeit eines „Fachtierarztes für Katzen“ zu warten. Da es aber Sinn macht, seine durch viele Mühen erworbene Expertise in einem bestimmten Fachgebiet zu dokumentieren, werden im Folgenden die 3 derzeit möglichen Wege zu einer solchen Dokumentation für praktizierende Tierärzte in Europa kurz angesprochen.

\section{ISFM}

Nach über 10 Jahren Diskussion hat die International Society of Feline Medicine (ISFM) vor einigen Jahren eine Vereinbarung mit dem Australian \& New Zealand College of Veterinary Scientists (ANZCVS) getroffen. So wird die dortige seit langem etablierte Prüfung in Katzenmedizin zur Aufnahme ins College (MANZCVS medicine of cats) auch 1-mal jährlich in Europa (in England und in englischer Sprache) durchgeführt. Nach Bestehen dieser durchaus anspruchsvollen Prüfung wird man zum Akademiemitglied der ISFM. Als gute Vorbereitung auf dieses Examen kann der (zeitintensive) Fernkurs „Feline Medicine“ des Centre of

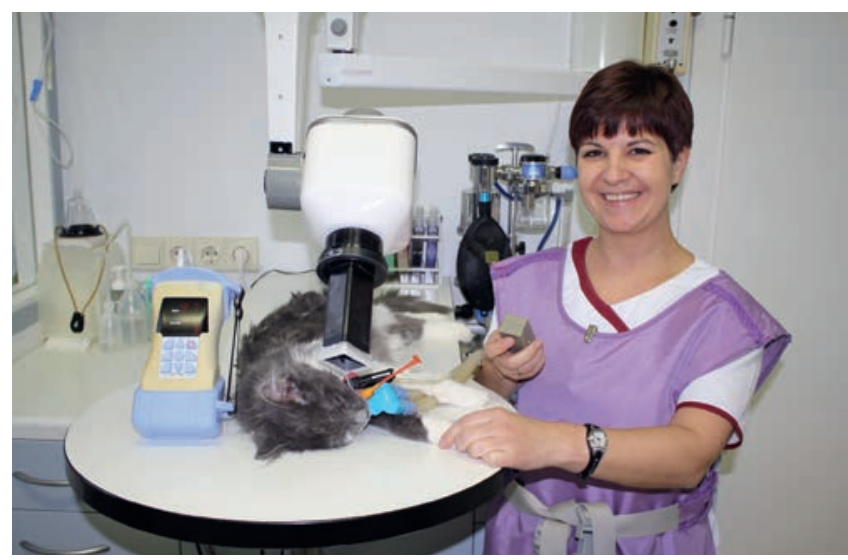

Abb. 3 Zahnröntgen ist ein wesentlicher Bestandteil der apparativen Ausstattung einer Katzenpraxis. (c) J. Kremendahl

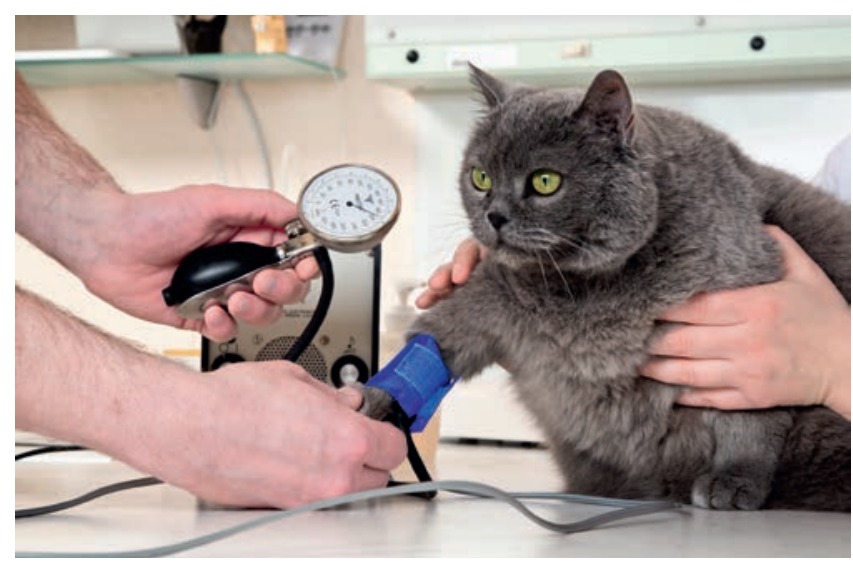

Abb. 4 Blutdruckmessung bei der Katze mittels Doppler. () J. Kremendahl 
Veterinary Education (CVE) in Sydney, Australien, gelten.

\section{ABVP}

Eine andere Möglichkeit besteht in den USA durch die Aufnahmeprüfung des American Board of Veterinary Practioners (ABVP) im Fachgebiet „Feline Practice". Auch hier handelt es sich um eine äußerst anspruchsvolle Prüfung, der man sich aber nur in den USA unterziehen kann. Während das australische Examen ein Tierärzte-Leben lang gilt, muss man sich dem amerikanischen Examen alle 10 Jahre erneut unterziehen, es sei denn, man hat im fraglichen Zeitraum genug relevante Publikationen produziert. Dieses Prinzip dürfte bei deutschen Fachtierärzten sicher nicht durchweg Begeisterung auslösen.

\section{ESVPS}

Schließlich, um auf die einzige deutschsprachige Möglichkeit einer solchen Dokumentation zu kommen, gibt es die European School of Veterinary Postgraduate Studies (ESVPS), Yelverton, Devon, England. Hier steht das „General Practioner Certificate in Feline Practice ${ }^{\text {" }}$ zur Verfügung. Es gibt einen Anbieter von tierärztlichen Fortbildungen in Deutschland, der einen Modulkurs zur Vorbereitung auf diese Prüfung durchführt.

\section{Fortbildung}

Auch wenn es inzwischen erfreulich viele Katzenthemen wie auch ganze Vortragsstränge zum Thema Katze auf deutschsprachigen Fortbildungsveranstaltungen und Kongressen gibt, finden fast alle großen internationalen Treffen - wie der oben erwähnte Katzenweltkongress in Barcelona - in englischer Sprache statt. Leider besteht diese sprachliche Hürde auch, was den Zugang auf wesentliche Literatur betrifft, auf Fachbuch- wie auf Fachzeitschriftenebene. In jedem Fall ist hier das „Journal of Feline Medicine and Surgery“ der ISFM zu nennen, das seit 1999 monatlich erscheint. Es wechseln sich „klassische“ Ausgaben mit Forschungsarbeiten und Fallberichten und „klinische“ Ausgaben mit hervorragenden Beiträgen für den Praxisalltag ab. Alle Ausgaben sind auch elektronisch verfügbar.

\section{Ausblick}

Der Trend zur „Katzenpraxis“ in Deutschland wird sich - wenn auch im Vergleich zu anderen Ländern weiterhin zeitverzögert - fortsetzen. Dieser Überzeugung bleibt der Verfasser auch nach 2 Jahrzehnten treu. Manchmal wundert er sich allerdings ein wenig über die eigentümliche und bedächtige Dynamik dieses Prozesses hierzulande. Hat Jeannie, die amerikanische Katzenpraktikerin, vielleicht nicht ganz unrecht, wenn sie schmunzelnd vermutet: „Mit Kreditkarten und Automatikgetrieben habt Ihr Euch doch auch lange schwer getan, aber irgendwann ...!"?

Die Notwendigkeit ergibt sich aus vielen nahe liegenden Aspekten. Immer mehr Menschen leben anders als noch vor einigen Jahrzehnten: Single-Dasein, Urbanisierung und Gentrifizierung, um nur einige zu nennen, sind allgegenwärtige Entwicklungen, die unabhängig davon, ob man sie begrüßt oder nicht, die Eignung der Katze als Haustier stärken. Ob das der Katze immer gut bekommt, bleibt abzuwarten. In der Praxis des Verfassers nehmen Verhaltensstörungen - bei durchaus gemischtem Umfeld - stark zu. Auch hier wird der Bedarf an Spezialisten deutlich zunehmen.

Die Katze, besonders auch die „gentrifizierte“ Katze, lebt fast doppelt so lange wie noch vor 25 Jahren. Sie wird immer älter und kränker, hat es an den Nieren, der Schilddrüse und den Knochen. Es handelt sich oft also um Erkrankungen, die bei Erhaltung der Lebensqualität gut von uns kontrolliert werden können. Die dazugehörigen - in der Regel anspruchsvollen - Besitzer möchten eine kompetente Behandlung und eine angemessene Kommunikation.

Der letzte, bewusst etwas überspitzt formulierte Absatz soll keineswegs von der „Tierschutzkatze“ ablenken, die im gleichen Maße unserer Hilfe bedarf. Katzenschutzorganisationen leisten mit großem Einsatz wertvolle Beiträge zur Reduktion des allgegenwärtigen Katzenelends. Oft könnte jedoch noch mehr zum Wohl der Schützlinge erreicht werden, wenn gewisse Maßnahmen in Absprache mit einem Tierarzt, der sich mit Populations- 
medizin und Infektionskrankheiten der Katze unter Tierschutzbedingungen auskennt, erfolgen würde. Immer wieder kommt es zu unnötigen Ausbrüchen von Infektionskrankheiten wie Panleukopenie mit zahlreichen Todesfällen, weil elementares Wissen fehlt und alte „Glaubenssätze“ („Das haben wir immer schon so gemacht ...“) nicht abgelegt werden können. Leider wird der Gedanke des Tierschutzes auch häufig von persönlichen Neurosen überlagert. Krasse Fälle von Animal Hoarding, z. B. mehrere Hundert Katzen in erbärmlichem Zustand in einem verlassenen Flugzeughangar in den Vereinigten Staaten, nehmen nicht nur jenseits des Atlantiks zu. Der Tierarzt ist also gefragt, seine Augen offen zu halten - in alle Richtungen.
Auch was diesen Aspekt betrifft, schafft es der ISFM vorbildlich, alle Bereiche der Katzenmedizin unter einen Hut zu bekommen und uns mit erstklassigen Informationen zu versorgen.

Der Verfasser möchte darauf hinweisen, dass sich in diesen Monaten die „Deutsche Gruppe Katzenmedizin“ in der Gründungsphase befindet und bereits mit der Organisation einer Katzenfortbildung mit namhaften Referenten begonnen hat.

Es handelt sich um Tierärzte, die sich in besonderem Maße der Katzenmedizin verschrieben haben und als nationaler Partner die Gedanken und das Wissen der ISFM weitergeben möchten. Spezielle Fortbildun- gen und andere Aktivitäten sind in der Planung. Engagierte Mitarbeit ist willkommen.

\section{Organisationen}

Anbei eine Übersicht über die verschiedenen Organisationen und deren Internetpräsenz (Stand: August 2015):

- American Board of Veterinary Practioners: http://www.abvp.com

- Australian \& New Zealand College of Veterinary Scientists: http://www. anzcvs.org.au

- Centre of Veterinary Education: http:// www.cve.edu.au/

- Deutsche Gruppe Katzenmedizin, nationaler Partner der ISFM: http:// germancatgroup.de

- European School of Veterinary Postgraduate Studies: http://www.esvps.org

- International cat care (Informationen zur Zertifizierung „cat friendly clinic“): http://icatcare.org/catfriendlyclinic

- International Society of Feline Medicine, ISFM: http://icatcare.org/vets

Online zu finden unter

http://dx.doi.org/10.1055/s-0035-1558512

\section{Literatur}

1 Bonaduce A. Sulla pleurite infettiva del gatto. Nuova Vet 1942; 21: 32-36

2 Graham JEB. An outline of feline medicine. Can Vet J 1961; 2 (7): 257-260

3 Sokolowski JH. Kleintierpraxis ausschließlich für Katzen. Waltham International Focus 1991; 1 (2): 16-20

Dr. Jürgen Kremendahl

MACVSC (medicine of cats) MRCVS

Berghauser Str. 128

42349 Wuppertal

praxis@dr-kremendahl.de 